\title{
An Experimental Case Study on Edge Computing based Cyber-Physical Digital Service Provisioning with Mobile Robotics
}

\author{
Daniel Pakkala, Jani Koivusaari, Pekka Pääkkönen \\ VTT Technical Research Centre of Finland Ltd. \\ firstname.lastname@vtt.fi
}

\author{
Jim Spohrer \\ IBM Research - Almaden \\ spohrer@us.ibm.com
}

\begin{abstract}
Digitalization of physical interaction and infrastructure intensive industries provides an opportunity for new kind of value co-creation via cyber-physical digital service provisioning. The rapid technological progress is enabling re-distribution of both physical and cognitive tasks and work between people and machines. The paper explores concept of cyber-physical digital service and presents an experimental case study on cyber-physical digital service provisioning for building diagnostics, utilizing a mobile robot as service actor and resource. The case study applies Design Science Research Methodology (DSRM) with an objective to identify insights on design challenges and digital technology infrastructure requirements of cyber-physical digital service provisioning. Based on evaluation of designed, developed and demonstrated trial system, insights on identified design challenges and related requirements for evolution of digital technology infrastructure are provided as results.
\end{abstract}

\section{Introduction}

Two parallel change drivers, servitization [1] and digitalization [2], are ongoing on societal level. These are changing how business processes, workflows and interaction takes place between legal entities in society. Here servitization refers to the ongoing shift from value-in-artifact paradigm towards value-in-use paradigm. Servitization is transforming organizations increasingly towards service provisioning in addition to products offering. At the same time, digitalization is transforming the business models, business processes and workflows to optimize human-machine cooperation, with a target of maximal exploitation of the capabilities enabled by new digital technologies. The ultimate crossing point of these two parallel drivers fully automated scalable service provisioning - is an attractive goal for many companies.
Many physical assets and interaction independent industries (e.g. media and finance) have been pioneers in digitalization and digital service provisioning (e.g. on-line banking, streaming video and music service offerings). More physical infrastructure and interaction dependent industries (e.g. construction, manufacturing, energy and facilities management) are transforming at considerably slower pace due to physical dependencies in business models, business processes and workflows. However, the rapid progress in Internet-of-Things (IoT) [3], Cyber-Physical Systems (CPS) [4], automation [5], robotics [6] and Artificial Intelligence (AI) [7] is enabling increasingly complex social and physical tasks to be automated. As presented in [8], the progress in these technologies is reshaping also service provisioning by changing work and tasks allocation in service between human labor and advanced technical systems. These systems are embodied in many different forms and have been collectively defined as service robots in [9]. On this new socio-physical frontier of digitalization, work is being automated and augmented via introduction and use of different kinds of automation and robotic systems (e.g. cyber-physical production systems [10] and service robotics [11]), which interact directly with people and the physical environment to create desired and designed outcomes and value.

In the manufacturing sector, the degree of automation has steadily increased in factories. On other sectors, automating physical and cognitive tasks and work is also becoming an interesting opportunity via cyber-physical digital service. This paper presents an experimental case study on such an opportunity in the context of digital transformation of facilities management. A cyber-physical digital service relying on mobile robotic for measurements and data collection in building diagnostic service is experimented.

Service is highly situated and performative value co-creation phenomena emerging as result of interaction between service actors and resources (both human and artefact) within a service system [12], [13]. 
Accordingly, in context of cyber-physical digital service, the artefact service actors and computing resources involved in the value co-creation become of interest in order to be able to design, develop and operate cyber-physical digital service offerings.

Fog/edge [14] computing has recently become a focal point of computing research complementing the current cloud computing dominant computing landscape for cyber-physical digital service provisioning by providing computing resources nearby the physical point of interest (e.g. a building). Fog/edge computing, from now on referred as edge computing in this paper, is a potential enabler for distributed computing applications requiring low/guaranteed latency, reliability and high bandwidth [15], [16] and [17]. Due to these characteristics differentiating from cloud computing, edge computing may hold potential as an enabler for implementation of IoT, CPS, automation systems and distributed robotics applications. Accordingly, it can be seen as an emerging computing infrastructure enabler for provisioning of cyber-physical digital service based on application of these technologies. Edge computing is facing a kind of 'chicken or egg' problem - no standard or widely available provisioned edge computing infrastructure exists, so no cyber-physical digital service offerings can be designed and provisioned relying on it. Current provisioning of cyber-physical digital service is based on service and customer specific IoT/CPS/automation system implementations, which may lead to challenges in customer enterprise IT architecture and data management. This is mainly due to resulting parallel service specific subsystems, which all need to be integrated, interoperable and managed as part of the enterprise architecture [18]. Motivated by this problem, this paper presents a design science research methodology based case study, where both a cyber-physical digital service offering and a supporting edge computing infrastructure are designed, developed and demonstrated in parallel with a research objective to gain insights into challenges and opportunities in edge computing based cyber-physical digital service provisioning.

A recent paper [19] conceptualized and proposed definition for digital service in context of service science and service systems, where digital service was defined as an autonomous technical system-of-systems, which represent technological agency of the service provider in value co-creation interaction with service user/customer. This viewpoint on digital service, defining it as a technical system-of-systems of Information, Communication, Computing and Automation Technologies (ICCAT) artefact, covers also IoT and CPS type of systems as enabling technical subsystem types for realization of digital service. For avoidance of doubt, the term digital service in this paper is to be understood as defined in [19].

Whereas the cyber-physical interface is in many ways present in service and service engineering, this paper focuses especially in identifying insights, design challenges and digital technology infrastructure requirements of cyber-physical digital service provisioning. This is approached via an experimental case study and trial system on cyber-physical digital service provisioning, including a mobile robot and its capabilities representing the technological agency of digital service provider in value co-creating interaction. In the case study, the cyber-physical interface is evident from two main viewpoints: 1) cyber-physical actuation, where a mobile robot participates in value creating interaction in the physical world for value cocreation with the service customer. 2) the overall value proposition of the experimental digital service is related to management of physical assets (building condition monitoring to support facility management). It is worth pointing out that in the case study (as well as in many other IoT and CPS based systems and service offerings) the value co-creating interaction is not only related to interaction between service system actors, but includes interaction also with the physical world (environment and artifacts/objects).

Technological progress is enabling cyber-physical digital service offerings, where value can be co-created with customers increasingly also in physical world related needs and interaction. As pointed out in [20], service systems engineering faces the challenge to bridge the boundaries of tangible and intangible resources, referring to data and automation, in new service systems innovations. This is especially relevant challenge for cyber-physical digital service provisioning, where the tangible physical world objects are subject to sensing, monitoring, analysis, decision making and actuation via intangible data-driven software intensive distributed and complex systems. These are almost intangible manifesting themselves only as written computer programs, collection of sensors, computing devices and possible actuators/robots in the physical world.

Cyber-physical digital service provisioning refers to value proposition of the service being related to physical word objects and locations, as well as to IoT, CPS, automation or robotic systems present as part of the technical provisioning system. The cyberspace centric sensing-analysis-actuation framework of this type of service provides an interesting interface to research fields of agent based, autonomic and AI systems. As proposed in [20], AI or machine intelligence (intelligence demonstrated by machines/artifacts) in combination with human intelligence allows new forms of resources integration 
and service provision in service systems. In [20] the authors also call for more research on re-configuration of service components and resources in service systems. Section 2 of the paper explores the relation of AI and cyber-physical digital service in the context of digitalization and service systems.

The rest of the paper proceeds as follows. Section 3 presents the DSRM [21] based experimental case study on cyber-physical digital service provisioning, followed by section 4 describing the trial system and demonstration. Section 5 provides discussion on results followed by concluding remarks and description of future research directions in section 6 .

\section{Role of AI and Cyber-Physical Digital Service in Digitalization}

At the core of digitalization is the target of new value creation via exploiting capabilities of digital technologies. In organizational and industrial context, this value realizes only via adoption and use of advanced technical systems as part of the activity, which is under digitalization. In the same context, the activities of interest are typically employee roles, tasks and business processes of the organization. The activities include both cognitive and physical tasks for actors that are participating in those. The actors participating the activities may include varying configurations of people (e.g. employees) with tools (e.g. hand tools and information systems) and intelligent machines (e.g. mobile robots, software robots and chat bots). Regarding progress in AI technology, the new capabilities can be exploited as technical systems to increase cognitive capabilities of both the actor types: people (augmented intelligence) and machines (machine intelligence). Regarding cyberphysical digital service in organizational context, the value co-creation interactions may take place between people and machine actors, or between machine actors, of two different service system participant organizations (legal entities of a B2B service). If the actors co-creating value are under governance of the same legal entity, the case can be considered as internal service provisioning. However, no value is cocreated on organization-to-organization level in that case. To summarize, digitalization can be viewed as service system engineering, where also advanced technical systems with autonomous decision-making and interaction capabilities are considered both as actors and resources within a service system. Examples of these kind of technical systems include AI systems and autonomous IoT/CPS/automation and robotic systems.
In organizational context, digitalization is about continuous re-organization and re-distribution of roles, tasks and responsibilities between the human and intelligent machine actors in work, based on evolution of capabilities of both. When intelligent machine actors participating in organizational workflows are provisioned 'as a service' by a different legal entity from the workflow owner, the setting can be viewed as digital service. When the workflow participated by an intelligent machine actor includes interaction with physical environment, objects or artifacts, the case can be characterized as cyber-physical digital service. Accordingly, building on a previous definition of digital service in [19], we define cyber-physical digital service as follows: a service executed by operationally autonomous ICCAT based system co-creating value for service user via interaction with physical environment, objects or artefacts in spatiotemporal situated context.

A noteworthy difference in cyber-physical digital service, when compared to digital service in general, is that value co-creation between service system entities is primarily based on physical world interaction of service actors and resources in spatiotemporally situated context. Also service user is not necessarily the initiator of the value co-creating service interaction, but the interaction may also be trigger based on changes in the physical spatiotemporal context perceived by the operationally autonomous ICCAT based system as a service actor. Examples of this type of cyber-physical digital service offerings include e.g. driving security and assistance features in cars and automated natural disaster alerts.

Progress in AI technology increases cognitive capabilities of both, the tools targeted for augmenting human actors at work, as well as intelligent machine actors at work. When combined with the continuous nature of digitalization, industrial organizations are facing a need to adopt new methods for analyzing optimal tasks and role allocation between humans and machines co-operating in workflows and business processes. These methods should allow analysis of cognitive and physical requirements of individual actor roles and tasks on such level of detail, which allows reorganization and re-allocation continuously between the two actor types. The feasibility of re-organization and re-allocation of roles and tasks between humans and machines is dependent on availability of continuously evolving digital technology capabilities.

The increasing cognitive capabilities and autonomy of intelligent machines at work provide interesting opportunities for cyber-physical digital service provisioning. The main categories of opportunities for cyber-physical digital service in this context include:

1) Human augmentation: Augmenting human employee's physical and cognitive capabilities in their 
existing workflow or business process role and tasks. For example service offerings for monitoring, diagnostics and remote operation of physical assets, objects and machines.

2) Machine augmentation: Fully digitalizing physical and cognitive tasks, workflows and roles within organization business processes via advanced cyber-physical cognitive technical systems. For example mobile service robotics and fully automated manufacturing systems.

The experimental case study presented in this paper belongs to category of machine augmentation and explores design challenges related to use of machine learning and mobile robotic components in cyberphysical digital service provisioning.

\section{Case and Service Scenario Description}

The purpose of the case study is to gain insights to design challenges and technology infrastructure requirements of cyber-physical digital service provisioning via experimentation in the current technological context, where no standard reusable technology infrastructure exists for supporting design, development and provisioning of cyber-physical digital service offerings. The societal and technological context and relevancy of cyber-physical digital service were described in sections $1 \& 2$ of the paper.

The case study was done within a research project studying provisioning of edge computing infrastructure for application development with 20 experienced researchers participating from the fields of computing, communication, information systems and software engineering. An experimental system was designed, developed and demonstrated for automated building measurements data collection as an enabler for digitalized facility management service provisioning. The experimental system was designed in an envisioned service system context including building owner as service customer and building diagnostics service provider, with additional role of digital infrastructure provider introduced as service resource owner (edge computing resources), and as facilitator in cyber-physical digital service provisioning. The system was demonstrated at realistic office building environment. Focus group discussions within the project team was applied as evaluation methodology throughout the design, development and demonstration phases of the project.

The DSRM applied in the case study has been described in [21]. Regarding evaluation within the DSRM process we refer to [22], further refining the evaluation activities to ex ante and ex post evaluations within the DSRM process. DSRM includes six interlinked activities, which are summarized below for the case study presented:

1. Problem identification \& motivation: Digitalization and automation of data collection in building diagnostic service, motivated by potential efficiency increase of avoiding experts performing data collection manually at the customer sites in building diagnostic service provisioning.

2. Defining objectives of a solution: The objective is to design a system capable of automating building measurements data collection at different customer sites not known at the design time.

3. Design \& development: A mobile robot is used as a service front line actor for collecting the data needed for experts to analyze the data and diagnose the building. As measurements and data collection physically at customer buildings is a generic and repeating task in building diagnostic service provisioning, the problem is approached from the general utility viewpoint. Mobile robot should act as semi-autonomous service actor navigating safely in a customer building with required and changeable sensors on-board for measurement data collection. The experimental system was designed and developed as two complementary prototype implementations: 1) A prototype of mobile robot based data collection for building diagnostics. 2) A prototype of edge computing and communication infrastructure designed for supporting and facilitating of cyber-physical digital service provisioning (a service resource). The separation allowed analysis and categorization of functionalities into service specific functionalities (part of prototype 1) and generally needed functionalities for cyber-physical digital service provisioning (part of prototype 2).

4. Demonstration: The trial system was demonstrated in a real building environment with a test use case of avoiding situations where the robot navigates through crowded areas in the building. This required real-time information exchange and interoperability between the service provider technical systems (mobile robot) and service customer technical systems (crowded areas detection in a building). The use case was selected to get further insights on design challenges and digital technology infrastructure requirements related to situated and spatiotemporal interaction and interoperability requirements between service system actors in cyber-physical digital service provisioning.

5. Evaluation: The evaluation was done based on evaluating the operationality, implementation feasibility and related design challenges of both prototypes. The evaluation is a combination of ex ante evaluation (during prototypes design \& development) 
and ex post evaluation (after demonstration) and is summarized in section 5 of this paper.

6. Communication: The research, with identified insights on design challenges and digital infrastructure requirements of cyber-physical digital service provisioning as results, are described in this paper. Supporting video material on demonstration of the test use cases is also available as supporting material for communication of results.

To provide a context for design and development of the experimental system, a service scenario and stakeholders were defined for the case study: Facility management service, where the building owner has need for on-demand building diagnostics and acquires this as a service. Today this usually involves negotiating and making a consultation or service agreement with a building diagnostics expert, who does measurements, analyses those and reports the results to the customer (e.g. on the health of structures). The case study addressed here explores the digitalization potential of the aforementioned consultation/service offering by exploiting a sensor equipped mobile robot as a semi-autonomous service front line actor. The service scenario includes also human actors as employees of the involved organizations. Value proposition of the service is fulfilled via a series of complementary interactions, including human-to-human, human-to-machine, environment-to-machine and machine-to-machine interaction. A special robotic mediation related concern and requirement for the service is that the robot should not enter crowded areas of the building for safety and measurement quality reasons. Accordingly, the service customer provides real-time dynamically changing headcount information per area within the building for the service provider. A Digital Infrastructure Provider role, providing a widely available distributed edge computing and communication environment for facilitating cyber-physical digital service provisioning is assumed in the case (not existing today).

The service system modeling of the case service scenario is presented in Figure 1. The figure illustrates the value proposition and service scenario of interest with the involved service system participants (organizations - business-to-business (b2b) type of service). Governed by policies, processes and management of each involved organizational participant, also the related service system actors and resources are illustrated. Furthermore, the value cocreating interactions between service system resources and actors in the experimental case are illustrated. It is worth pointing out the fact that in value co-creation of business-to-business type of service, the organizations are considered the as service system entities and value co-creation is expected to be realized on the organizational level according to the value proposition, giving the basis for engaging in service interaction. However, as organizations are not physical world actors as such (but are governed structures of actors and resources), the value co-creation in b2b service realizes only via service interaction between actors (human or technological) and resources of two or more different legal entities (provisioning of external service). The value co-creation interaction among actors and resources of a single organization is considered internal service provisioning and is out of the scope of the case and this work.

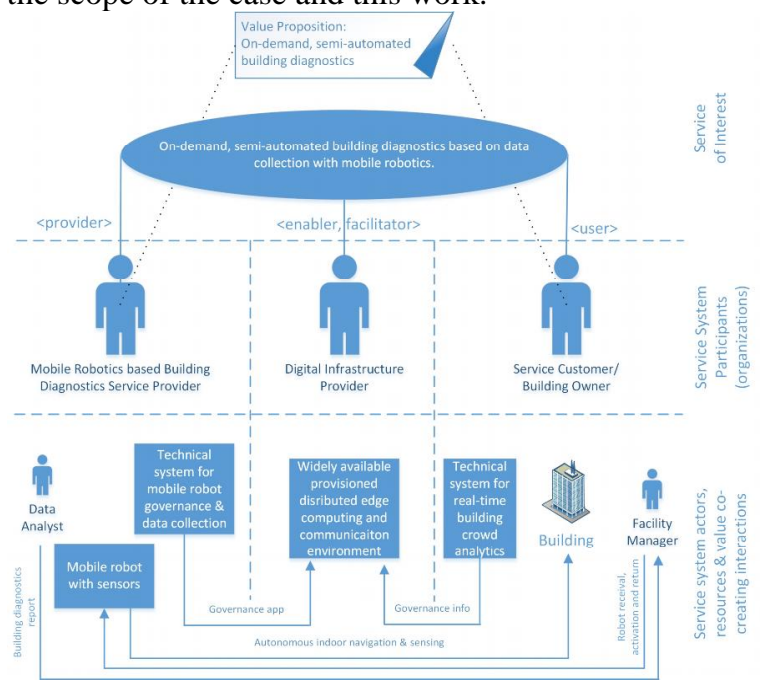

Figure 1. Case service scenario illustration.

A summative service scenario between the related organizations and actors is described below (relation to the trial system prototypes and the demonstration indicated in parenthesis):

1. On behalf of a building owner, a facility manager makes a service order/contract at the webpage of a service provider based on the service description and the value proposition of the service (standard web technology - not implemented in the experimental system)

2. The service provider ships a mobile robot with on-board sensors to the customer address and deploys a robot governance app on edge computing and communication infrastructure of a digital infrastructure provider. (Management and provisioning of edge computing and communication infrastructure Prototype 2)

3. The facility manager receives the robot and activates it for autonomous navigation and data collection within desired area of the building (Prototype $1+$ Demonstration in real environment).

4. The robot autonomously navigates between the set waypoints in the building collecting data with on-board sensors. The navigation is dynamic in a way 
that crowded areas of the building are avoided by autonomously finding alternative routes between waypoints when congestions in the building are detected (Prototype $1+$ Demonstration in real environment).

5. After the data collection is complete, the facility manager ships the robot back to the service provider. Service provider analyses collected data and delivers building diagnostics report to the facility manager. (not implemented in the trial system, but the related requirements for edge computing and communication infrastructure are considered in ex post evaluation)

\section{Experiments and Demonstration}

The experimental system and demonstration was implemented as two separate but complementary prototype systems. The separation allowed analysis, design and categorization of digital service functionalities into service specific functionalities (part of prototype 1) and generally needed support and functionalities for cyber-physical digital service provisioning (part of prototype 2 ).

Prototype system 1 is illustrated in Figure 2. The system consisted of three main connected computing devices: 1) an application server in the role of Multiaccess Edge Computing (MEC) server hosting software components for message exchange with the connected devices, as well as for the application logic of dynamic and automatic robot governance based on congestion information within the building. 2) A site resident computing device with hardware acceleration for artificial neural network based computing (NVIDIA Jetson). The device was connected to a web camera and hosted software for the detection of the number of people from its video feed in real-time, as well as communicating this information forward. 3) A commercially available mobile robot (MiR100), which was used without modifications and was interacted with via the graphical user interface it provides for human instructors, as well as via the Application Programming Interface (API) for software based interaction. The robot was equipped with an additional 'on-board' LIDAR device for continuous data collection. Analysis of LIDAR data was not implemented, but the data was visualized as live streaming point cloud data in demonstration.

Regarding the software for the communication between the computing devices, MQTT was used in message exchange between devices $1 \& 2$. HTTP was used between devices $1 \& 3$. Python was used as programming language in software component implementations. Regarding network connectivity between devices $1 \& 2$, both $4 \mathrm{G}$ wireless broadband access and WLAN were tested. WLAN was used in the demonstration. Figure 2 also presents the computing devices governance between the three involved service system participant organizations. The participant organizations are assumed to govern their own computing resources as part of the digital service provisioning and governance.

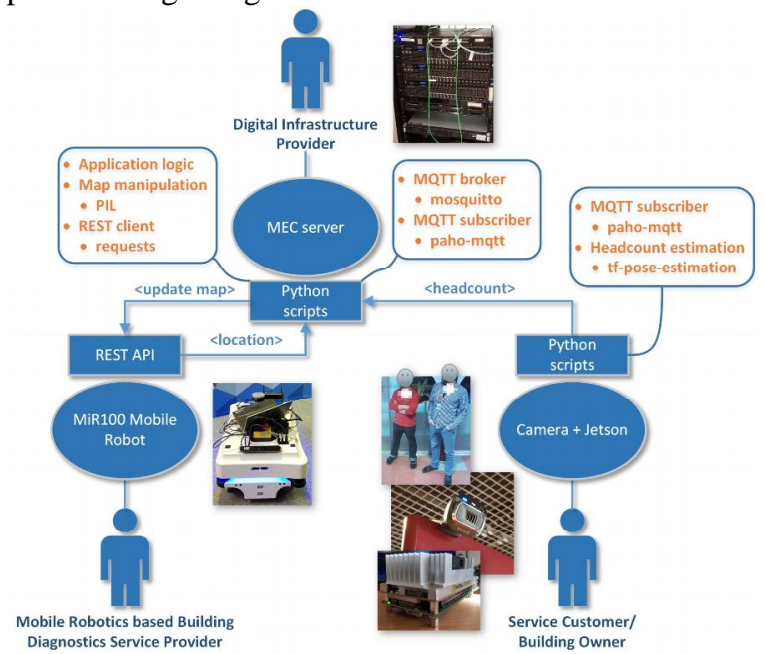

Figure 2. Prototype system 1 illustrated.

Prototype system 2 was created to experiment if the computing environment including cloud instance, edge computing server and on-site computing devices could be managed as horizontally integrated computing cluster in order to enable systematic management and provisioning of the distributed computing infrastructure for virtualized and modular software deployment of the service component implementations. Figure 3 illustrates prototype system 2 set-up with relation to involved service system participants of the case. In prototype 2 , the service components implemented as software are assumed to be packaged and managed as Docker images, and are provided by the digital service provider. The service component implementations, which are to be deployed are specified with Kubernetes Helm format, which includes references to the Docker images among other deployment information (e.g. service port and protocol). The service component implementations are deployed on Rancher v2.x platform based on the provided Helm specification, which is added to Rancher's service catalog via a Git repository (BitBucket). The Helm specification with referenced Docker images is used as a mean to define the needed service component implementation deployments within the computing cluster to set-up the cluster for digital service provisioning. The digital infrastructure provider governs the cluster and deployments of 
service component implementations within it. The service component implementations with descriptions are registered and provided to Service Component Registry by the Digital Service Provider.
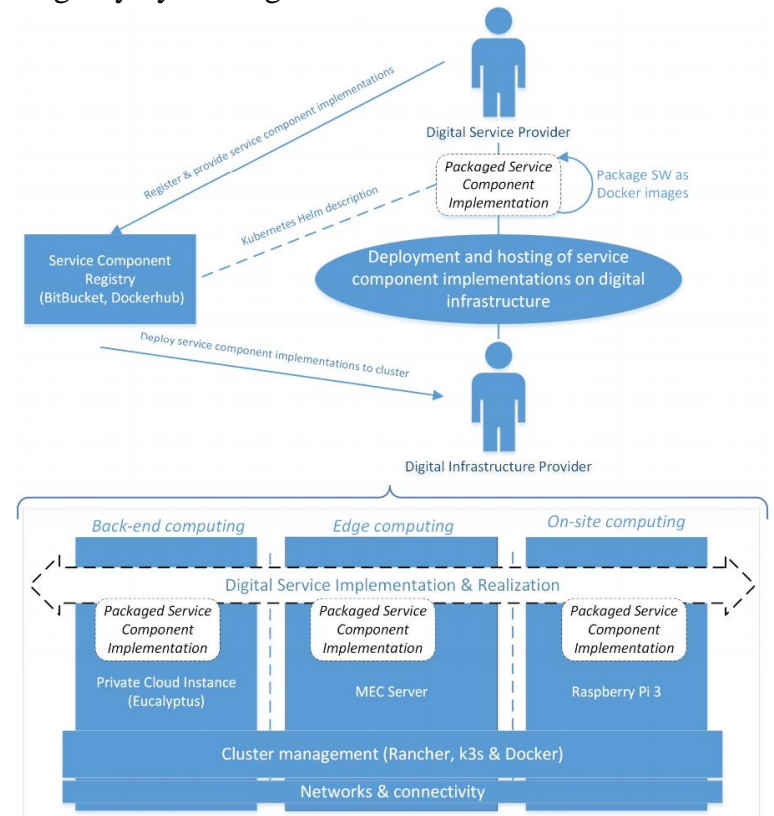

Figure 3. Prototype system 2 illustrated.

One lightweight Kubernetes cluster (k3s) was set up consisting of three computing nodes: a private Eucalyptus cloud instance, an application server in the role of MEC server and Raspberry Pi 3 device as onsite edge computing node. The cluster was registered to Rancher to enable deployment of service component implementations within the computing nodes of the cluster. Ideally, the software component implementations of prototype 1 could have been deployed and demonstrated within the computing cluster set up in prototype 2, however this was not tested or demonstrated in the trial system. Design, development and testing of prototype 2 resulted to identification of further technological design and research challenges described in section 5 .

In the demonstration the robot was executing its data collection mission in the building by navigating between route points set by the robot instructor (e.g. facility manager). Two variant use cases were demonstrated: 1) The robot performing its service related mission and navigating its set route without congestion. 2) The robot performing its service related mission, but a congestion is detected at its navigation route via customer systems, creating a need for externally re-routing the robot before it enters the congested area. Figure 4 illustrates the birds eye view to the demonstration of the test use case for both use case variants, as well as the floorplan of the test site equipped with real-time video analytics based crowd detection subsystem. In the figure, the route of the robot is illustrated with blue dashed lines between the route points set by the robot instructor (blue circles).

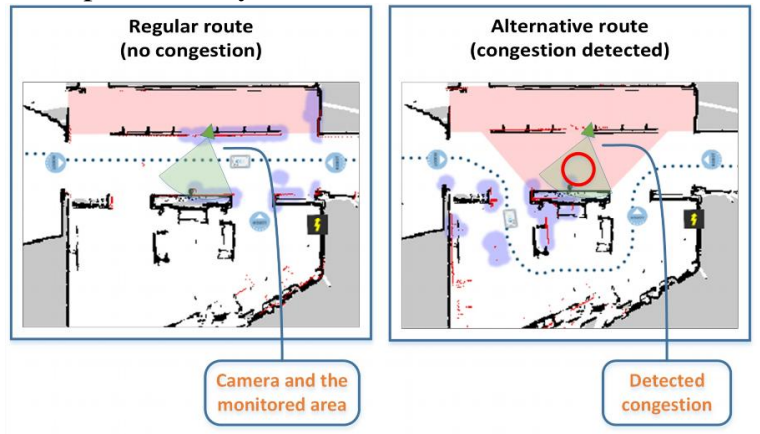

Figure 4. Demonstration view with regular and alternative route to avoid congested area.

The congestion detection and external re-routing of the robot via its APIs were implemented by dynamically modifying the 'forbidden areas' (pink areas in Figure 4) of the robot's internal map, which it uses for navigation. Accordingly, the exact new route is not given for the robot, but only a forbidden area is indicated in the manipulated map, matching the area where congestion is detected. The robot's autonomous navigation feature finds a new route using the manipulated map for route selection. When congestion is detected the robot's mission is stopped, the map is updated and the mission resumed. The overall external intervention to the robot's mission causes a delay of few seconds before the mission is resumed.

\section{Discussion on Results}

Whereas the existing studies on role of AI and robotics in service [8], [9] have focused on conceptual level exploration, this work contributes an experimental case study with a trial system in a laboratory setting revealing new insights on challenges related to design and provisioning of cyber-physical digital service with mobile robot and AI components.

To increase understanding on cyber-physical digital service as a highly situated and performative phenomena of interaction between service actors and resources, a definition for cyber-physical digital service is proposed in the paper with a discussion how it is rooted in fundamentals of service science and a previous definition of digital service.

The demonstration of the experimental system in a real environment indicates implementation feasibility of mobile robot and AI components based autonomous actuation in cyber-physical digital service provisioning. In the evaluation of the experimental 
system insights on design challenges and digital technology infrastructure requirements were identified. As a key insight, the spatiotemporal interaction and interoperability requirements for value co-creation between multiple cyber-physical digital service providers towards the same service customer is worth of further research. The spatiotemporal interaction and interoperability requirement is identified as one of the key differentiators in the design of cyber-physical digital service, when compared to digital service targeted for value co-creation purely in the cyberspace. In cyberspace the time and place rarely cause central requirements for digital service. In cyber-physical digital service provisioning the spatiotemporal interaction requirements between service actors and resources, as well as performance of those, become central for the design of value proposition and service interaction. In our case the service provider and customer technical systems needed to exchange information in real-time as a response to dynamically changing crowd conditions in the building. Furthermore, the mobile robot as a physical world actor needed to adapt its behavior dynamically in realtime, as a response to changing crowd conditions in the building.

As evaluation results, findings on design challenges (DC) and digital infrastructure requirements (DIR) from prototypes $1 \& 2$ evaluation include:

- The trial system required setting up a network for the robot's connectivity, message exchange subsystem for message and event exchange, and a piece of software application logic for dynamic robot re-routing. If these would be available as reusable offerings (micro-services) on a provisioned digital infrastructure, the design, development and provisioning of the service would be considerably facilitated (DIR\#1: Micro-service offerings for connectivity, message exchange and event management).

- A shared coordinate system for managing the dynamic situation related information flows within buildings was found as an interesting design challenge related to enabling spatiotemporal interaction and information exchange needs between the service actors and resources (DC\#1: Shared coordinate system for spatiotemporal information exchange between service actors).

- More advanced autonomic capabilities of the robot would decrease customer responsibilities and skill requirements in receiving and activating the robot for mission. (DC\#2: Design of autonomous features and machine intelligence in cyber-physical service to minimize customer responsibilities and skill requirements for service use.
- Data streaming, storing and preprocessing at the edge would allow real-time data analytics and improve the value proposition of the service. (DIR\#2: Micro-service offerings for data streaming, storing and preprocessing). LIDAR data streaming was tested but it caused critical congestion to the whole local area network resulting in trial system failure. (DIR\#3: Network slicing and/or compression of point cloud data streams should be supported)

- The robot's missions had specific stages for reading external input via APIs. Operating the robot in a dynamic way proved to be challenging. The robot should provide APIs responsive during any state of a mission. (DC\#4: Design of autonomous workflows in a way that enables dynamic adaption to changes in physical context).

- Edge centric cluster management with capability to deploy containerized software and add front-end and on-site/in-device device computing nodes, as well as back-end cloud nodes can be implemented manually as an expert activity. Cluster management software provides also opportunities for deployment automation. However, provisioning and automated deployment management of horizontally integrated edge centric computing cluster presents a design challenge for scalable provisioning of distributed edge computing infrastructure. The subchallenges include design of digital service provider facing digital service description format, service component registry and deployment automation in a way, which does not require deep understanding on specific technologies used for cluster implementation and management. (DC\#5: Scalable automated provisioning of distributed edge computing infrastructure for cyber-physical digital service software deployment and operation)

- The unified management of computing and communication resources within horizontally integrated distributed edge computing infrastructure is a research problem and design challenge in enabling scalable provisioning of edge computing infrastructure. (DC\#5: Unified management of computing and communication resources of distributed edge computing infrastructure towards digital service providers)

- The integration between lightweight Kubernetes cluster, Rancher 2.x, Docker Hub, and Git repository enabled deployment of service component implementations to MEC server and Raspberry Pi based on container images published in the Docker Hub. (DIR\#4: Provisioned edge computing infrastructure needs to support repository and specified description language for service component software implementations management between a digital service provider and the digital infrastructure provider) 
- $\quad$ Service implementation management platform (Rancher) deployment in public domain was tested, but $\mathrm{k} 3 \mathrm{~s}$ cluster could not be registered to Rancher due to the firewall in the mobile domain. OpenVPN was experimented as a solution to the problem, but the connectivity problem remained unsolved. Also k3s couldn't be deployed successfully on the same node as Rancher due to interfering firewall configurations of the technologies. Thus, a separate VM was allocated for Rancher at the MEC server. (DC\#6: Design of secure automated network configuration as part of horizontal multi-network distributed computing cluster management).

Based on the design challenges and digital infrastructure requirements identified via evaluation of the case study, we summarize that even though mobile robotics based cyber-physical digital service provisioning can already be realized, there are many obstacles presenting future research problems, design challenges and digital infrastructure requirements before scalable and on-demand provisioning becomes feasible. Due to the inherent complexity of the technical systems realizing cyber-physical digital service, evolution of the digital infrastructure has central role in facilitating cyber-physical digital service provisioning. Currently the digital infrastructure between cloud computing based back-ends and the IoT/CPS/automation and robotic devices is highly heterogeneous requiring system engineering projects for realizing end-to-end cyber-physical digital service provisioning case-by-case. Accordingly, cost efficient, dynamic and scalable on-demand provisioning of cyber-physical digital service is not feasible in the current state of the digital infrastructure.

Edge computing as a field of R\&D has potential for introducing new innovations and improvements to the digital infrastructure for better supporting cyberphysical digital service design, development and operation. A well-designed and provisioned edge computing infrastructure could lower the cost of cyberphysical digital service provisioning considerably. This would require bringing the edge computing infrastructure provisioning to a comparable level with cloud computing (IaaS, SaaS, PaaS, FaaS, CaaS).

The design challenges and digital infrastructure requirements, identified as results of this work, give some insights to what is expected and needed from the future edge computing infrastructure from the viewpoint of cyber-physical digital service provisioning. As a key finding of the work, these needs include support for spatiotemporal on-demand interoperability in information exchange between the technical systems of involved service system participants. On the side of digital infrastructure, generally needed micro-service offerings (connectivity, message exchange, event management, data storing and apps hosting) for enabling this information exchange are identified as a possible approach to address the need.

\section{Conclusion}

The lack of computing and communication infrastructure support at the edge of network globally hinders new cyber-physical digital service innovations by introducing design, development and operation complexity, case-by-case system engineering and high development project costs. However, this 'hole' in the current digital infrastructure also presents and interesting opportunity for research, development and innovation on the global digital infrastructure, with evident need and market potential due to ongoing digitalization and increasing demand for cyberphysical digital service.

The paper presented an experimental case study on cyber-physical digital service provisioning with an objective to gain insights on related design challenges and digital technology infrastructure requirements. As a key insight, the need for spatiotemporal on-demand interoperability in information exchange between the technical systems of involved service system participants was identified.

The evaluation of the experimental case study identified six design challenges and four digital infrastructure requirements for cyber-physical digital service provisioning. These provide some insights for future $\mathrm{R} \& \mathrm{D}$ on edge computing and communication infrastructure in supporting cyber-physical digital service provisioning.

As a concluding remark, cyber-physical digital service provisioning with autonomous technical systems (e.g. mobile robots) holds great potential for digitalization of physical interaction and infrastructure intensive industries. However further research is needed on cyber-physical digital service engineering methods, architectures and provisioning of the related technology and computing infrastructure, in order to enable efficient, high quality and scalable design, development, operation and governance of cyberphysical digital service. Different ecosystem and cooperation architectures, as well as future digital enterprise architecture are relevant research directions to increase understanding and close the knowledge gap on efficient value co-creation with cyber-physical digital service.

\section{References}

[1] C. Raddats, C. Kowalkowski, O. Benedettini, J. Burton, 
and H. Gebauer, "Servitization: A contemporary thematic review of four major research streams", Ind. Mark. Manag., Elsevier, Apr. 2019.

[2] J. Reis, M. Amorim, N. Melão, and P. Matos, "Digital Transformation: A Literature Review and Guidelines for Future Research", Springer, Cham, 2018, pp. 411-421.

[3] J. Gubbi, R. Buyya, S. Marusic, and M. Palaniswami, "Internet of Things (IoT): A vision, architectural elements, and future directions", Futur. Gener. Comput. Syst., vol. 29, no. 7, Elsevier, pp. 1645-1660, Sep. 2013.

[4] E. A. Lee, "Cyber Physical Systems: Design Challenges", in 2008 11th IEEE International Symposium on Object and Component-Oriented Real-Time Distributed Computing (ISORC), IEEE, 2008, pp. 363-369.

[5] M. Vagia, A. A. Transeth, and S. A. Fjerdingen, "A literature review on the levels of automation during the years. What are the different taxonomies that have been proposed?", Appl. Ergon., vol. 53, pp. 190-202, Mar. 2016.

[6] B. Siciliano and O. Khatib, Eds., Springer Handbook of Robotics. Berlin, Heidelberg: Springer Berlin Heidelberg, 2008.

[7] S. Russell and P. Norvig, Artificial Intelligence A Modern Approach Third Edition. Pearson, 2010.

[8] M.-H. Huang and R. T. Rust, "Artificial Intelligence in Service”, J. Serv. Res., vol. 21, no. 2, SAGE, pp. 155-172, 2018.

[9] J. Wirtz et al., "Brave new world: service robots in the frontline", J. Serv. Manag., vol. 29, no. 5, Emerald Publishing Ltd., pp. 907-931, Oct. 2018.

[10] L. Wang, M. Törngren, and M. Onori, "Current status and advancement of cyber-physical systems in manufacturing", J. Manuf. Syst., vol. 37, Elsevier, pp. $517-$ 527, Oct. 2015.

[11] M. Decker, M. Fischer, and I. Ott, "Service Robotics and Human Labor: A first technology assessment of substitution and cooperation", Rob. Auton. Syst., vol. 87, Elsevier, pp. 348-354, Jan. 2017.

[12] S. L. Vargo and R. F. Lusch, "Service-dominant logic: continuing the evolution", J. Acad. Mark. Sci., vol. 36, no. 1, Springer US, pp. 1-10, Mar. 2008.
[13] P. P. Maglio, S. L. Vargo, N. Caswell, and J. Spohrer, "The service system is the basic abstraction of service science", Inf. Syst. E-bus. Manag., vol. 7, no. 4 (Spec. Iss.), Springer-Verlag, pp. 395-406, 2009.

[14] M. Chiang and T. Zhang, "Fog and IoT: An Overview of Research Opportunities", IEEE Internet Things J., vol. 3, no. 6, IEEE, pp. 854-864, Dec. 2016

[15] I. Hadžić, Y. Abe, and H. C. Woithe, "Edge computing in the ePC", in Proceedings of the Second ACM/IEEE Symposium on Edge Computing - SEC '17, ACM, 2017, pp. $1-10$.

[16] G. Pocovi, T. Kolding, M. Lauridsen, R. Mogensen, C. Markmller, and R. Jess-Williams, "Measurement Framework for Assessing Reliable Real-Time Capabilities of Wireless Networks", IEEE Commun. Mag., vol. 56, no. 12, IEEE, pp. 156-163, Dec. 2018.

[17] P. Skarin, W. Tarneberg, K.-E. Arzen, and M. Kihl, "Towards Mission-Critical Control at the Edge and Over 5G", in 2018 IEEE International Conference on Edge Computing (EDGE), IEEE, 2018, pp. 50-57.

[18] A. Zimmermann, R. Schmidt, K. Sandkuhl, D. Jugel, J. Bogner, and M. Mohring, "Evolution of Enterprise Architecture for Digital Transformation," in 2018 IEEE 22nd International Enterprise Distributed Object Computing Workshop (EDOCW), IEEE, 2018, pp. 87-96.

[19] D. Pakkala and J. Spohrer, "Digital Service: Technological Agency in Service Systems", in Proceedings of the 52nd Hawaii International Conference on System Sciences, 2019.

[20] T. Böhmann, J. M. Leimeister, and K. Möslein, "Service Systems Engineering”, Bus. Inf. Syst. Eng., vol. 6, no. 2, Springer Fachmedien Wiesbaden, pp. 73-79, Apr. 2014.

[21] K. Peffers, T. Tuunanen, M. A. Rothenberger, and S. Chatterjee, "A Design Science Research Methodology for Information Systems Research", J. Manag. Inf. Syst., vol. 24, no. 3, M. E. Sharpe, pp. 45-77, Dec. 2007.

[22] C. Sonnenberg and J. vom Brocke, "Evaluations in the Science of the Artificial - Reconsidering the Build-Evaluate Pattern in Design Science Research", in Lecture Notes in Computer Science, vol 7286, Springer, Berlin, Heidelberg, 2012, pp. 381-397. 